\title{
Solution Generating Theorems and Tolman- Oppenheimer-Volkov Equation for Perfect Fluid Spheres in Isotropic Coordinates
}

\author{
Apisit Kinreewong ${ }^{1, *}$, Petarpa Boonserm ${ }^{2}$ and Tritos Ngampitipan ${ }^{3}$ \\ ${ }^{1,2}$ Department of Mathematics and Computer Science, Faculty of Science, Chulalongkorn University, Bangkok, Thailand 10300. \\ ${ }^{3}$ Faculty of Science, Chandrakasem Rajabhat University, Bangkok, Thailand 10900 \\ *Corresponding author
}

\begin{abstract}
Despite the possibility of finding exact solutions to the Einstein field equations, there is another way to obtain new exact solutions without having to directly solve the Einstein field equations. This method is the so called "solution generating theorems". In the descriptive approximation of stars, we will bring these solutions to analyze the realistic stars. One of the popular assumptions is a perfect fluid sphere. The TolmanOppenheimer-Volkov (TOV) equation describes the internal structure of general relativistic static perfect fluid spheres, including the pressure and density profiles. In this paper, we find relative solution generating theorems that map perfect fluid spheres into perfect fluid spheres in isotropic coordinates. In addition, we study and develop new solutions for the TOV equation.
\end{abstract}

Keywords-component; General relativity; perfect fluid sphere; isotropic coordinates; TOV equation

\section{INTRODUCTION}

General relativity, also known as the general theory of relativity, defines the geometric property of spacetime. Einstein presented an equation that explains the fundamental interaction of gravitation called the "Einstein field equation", which is as following [1-4]

$$
G_{\mu \nu}=8 \pi G T_{\mu \nu},
$$

where $G_{\mu \nu}$ is the Einstein tensor and ${ }^{T_{\mu \nu}}$ is the stress energy tensor.

The Einstein field equation is a fully non-linear partial differential equation which, in general, cannot exactly be solved. Therefore, some assumptions must be made in order to reduce the complexity of the equation. This assumption is the perfect fluid sphere, which has simply been developed as idealized models of stars. We can use the perfect fluid constraint to build several new exact solutions for any relativistic static perfect fluid sphere [5].

In astrophysics, the Tolman-Oppenheimer-Volkov (TOV) equation describes the internal structure of general relativistic static perfect fluid spheres. The significance of the TOV equation constrains is to study the interior structure of perfect fluid spheres, which directly provides information about the pressure profile and density profile [3-4, 6-11].
At present, we use the "solution generating theorems" to solve for new exact solutions which can comfortably generate the class of several new perfect fluid spheres.

In particular, we investigate the solution generating theorems and the TOV equation in isotropic coordinates, using pure mathematical principles as a way of finding several new solutions.

In this paper, we find relative transformation theorems that map perfect fluid spheres into perfect fluid spheres in isotropic coordinates. These transformations may allow some relationship between previously perfect fluid spheres and new perfect fluid spheres. In addition, we study and develop new solutions for the TOV equation, thereby also directly giving information about the pressure and density profiles of general relativistic static perfect fluid spheres.

\section{SOLUTION GENERATING THEOREMS}

\section{A. Isotropic Coordinates}

Generally, there are now several coordinate systems of perfect fluid spheres. One of the well-known coordinates is isotropic coordinates. A significant characteristic of the isotropic coordinates is that the coefficients of radial and angular coordinates are equal. The metric takes form

$$
\mathrm{d} s^{2}=-\zeta(r)^{2} \mathrm{~d} t^{2}+\frac{1}{\zeta(r)^{2} B(r)^{2}}\left\{\mathrm{~d} r^{2}+r^{2} \mathrm{~d} \Omega^{2}\right\}
$$

where $\mathrm{d} \Omega^{2}=\mathrm{d} \theta^{2}+\sin ^{2} \theta \mathrm{d} \phi^{2}$. So we derive

$$
\begin{aligned}
& G_{\text {晻 }}=-2 B^{\prime} B \zeta^{2} / r+\left(B^{\prime}\right)^{2} \zeta^{2}-\left(\zeta^{\prime}\right)^{2} B^{2}, \\
& G_{\text {瓶 }}=G_{\text {獜 }}=-B^{\prime} B \zeta^{2} / r+\left(B^{\prime}\right)^{2} \zeta^{2}-B B^{\prime \prime} \zeta^{2}+\left(\zeta^{\prime}\right)^{2} B^{2} .
\end{aligned}
$$

\section{B. Ordinary Differential Equation}

In the elementary step of the approximation of stars, we use perfect fluid spheres to build and develop idealized models 
of stars. We also make use of the properties of perfect fluid spheres. So we obtain

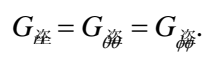

This equation is a "perfect fluid constraint" which supplies us with the ordinary differential equation [ODE] for any relativistic static perfect fluid sphere. Then we derive the ordinary differential equation for isotropic coordinates as [611]:

$$
\left(\frac{\zeta^{\prime}}{\zeta}\right)^{2}=\frac{B^{\prime \prime}-B^{\prime} / r}{2 B}
$$

We can write $g(r)=\frac{\zeta(r)^{\prime}}{\zeta(r)}$ in equation (6), that is

$$
g(r)^{2}=\frac{B^{\prime \prime}-B^{\prime} / r}{2 B} .
$$

Equation (7) can be rewritten in terms of $B(r)$ as:

$$
B^{\prime \prime}-\frac{B^{\prime}}{r}-2 g^{2} B=0
$$

\section{Solution Generating Theorems for Isotropic Coordinates}

As derived in [7], we have developed several "algorithmic" techniques that permit one to generate large classes of perfect fluid spheres. Let us now develop two solution generating theorems appropriate to isotropic coordinates.

Let's suppose that $\left\{\zeta_{0}(r), B_{0}(r)\right\}$ represents an initial perfect fluid sphere [8-9, 11].

Theorem 1. (7th BVW or Buchdahl transformation (T7)) Let $B_{0}(r)$ be fixed, then

$$
\mathrm{d} s^{2}=-\frac{1}{\zeta_{0}(r)^{2}} \mathrm{~d} t^{2}+\frac{\zeta_{0}(r)^{2}}{B_{0}(r)^{2}}\left\{\mathrm{~d} r^{2}+r^{2} \mathrm{~d} \Omega^{2}\right\}
$$

is also a perfect fluid sphere. That is, the mapping

$$
T_{7}:\left\{\zeta_{0}(r), B_{0}(r)\right\} \text { a }\left\{\frac{1}{\zeta_{0}(r)}, B_{0}(r)\right\} .
$$

takes perfect fluid spheres into perfect fluid spheres.

In addition, transformation theorem defined in theorem 1 is a "square root of unity" in the sense that $T_{7} \mathrm{o} T_{7}=I$, where $I$ is an identity. That is, when we apply theorem 1 twice, we obtain the initial perfect fluid spheres as the following

$$
T_{7} \mathrm{o} T_{7}:\left\{\zeta_{0}(r), B_{0}(r)\right\} \text { a }\left\{\frac{1}{\zeta_{0}(r)}, B_{0}(r)\right\} \text { a }\left\{\zeta_{0}(r), B_{0}(r)\right\} \text {. }
$$

After applying theorem 1 n-times, we can represent $\zeta(r)$ as:

$$
\zeta(r)= \begin{cases}\frac{1}{\zeta_{0}(r)} & \text { if } n \text { is odd number } \\ \zeta_{0}(r) & \text { if } n \text { is even number. }\end{cases}
$$

Theorem 2. (8th BVW transformation (T8)) Let $\zeta_{0}(r)$ be fixed and extend $B_{0}(r)$ to $B_{0}(r) Z_{0}(r)$. Define

$$
Z_{0}(r)=\left\{\sigma+\varepsilon \int \frac{r \mathrm{~d} r}{B_{0}(r)^{2}}\right\}
$$

for arbitrary $\sigma$ and $\varepsilon$. Then

$$
\mathrm{d} s^{2}=-\zeta_{0}(r)^{2} \mathrm{~d} t^{2}+\frac{1}{\zeta_{0}(r)^{2} B_{0}(r)^{2} Z_{0}(r)^{2}}\left\{\mathrm{~d} r^{2}+r^{2} \mathrm{~d} \Omega^{2}\right\},
$$

is also a perfect fluid sphere. That is, the mapping of

$$
T_{8}:\left\{\zeta_{0}(r), B_{0}(r)\right\} \text { a }\left\{\zeta_{0}(r), B_{0}(r) Z_{0}\left(B_{0}\right)\right\}
$$

takes perfect fluid spheres into perfect fluid spheres.

Definition 1. A transformation $T$ is called "idempotent" if $T$ oT @T where the symbol @ represents equality up to relabeling of the parameters. In this sense, any theorem is idempotent if we apply the transformation more than once, with no further solutions being obtained.

To see this, consider the mapping of theorem 2

$$
T_{8} \mathrm{o} T_{8}:\left\{\zeta_{0}, B_{0}\right\} \text { a }\left\{\zeta_{0}, B_{0} Z_{0}\left(B_{0}\right)\right\} \text { a }\left\{\zeta_{0}, B_{0} Z_{0}\left(B_{0}\right) Z_{1}\left(B_{1}\right)\right\} \text {. }
$$

When we apply the transformation of theorem 2 a second time, it does not lead to a new yield (no additional information). Therefore, theorem 2 is idempotent.

Having now found two solution generating theorems, this leads to a new corollary.

Corollary 1. Let $\left\{\zeta_{0}(r), B_{a}(r)\right\}$ and $\left\{\zeta_{0}(r), B_{b}(r)\right\}$ be a perfect fluid sphere and let $\zeta_{0}(r)$ be fixed. Then for all $q$,

$$
\left\{\zeta_{0}, q B_{a}+(1-q) B_{b}\right\}
$$


is also a perfect fluid sphere. Furthermore, all perfect fluid spheres for a fixed $\zeta_{0}(r)$ can also be written in this form.Proof for Corollary 1. From the equation in terms of $B(r)$, written as

$$
B^{\prime \prime}-\frac{B^{\prime}}{r}-2 g^{2} B=0
$$

We get $B_{a}^{\prime \prime}-\frac{B_{a}^{\prime}}{r}-2 g^{2} B_{a}=0, B_{b}^{\prime \prime}-\frac{B_{b}^{\prime}}{r}-2 g^{2} B_{b}=0$.

Assume that $\left\{\zeta_{0}(r), B_{0}(r)\right\}$ solves equation (16), we want to know if $\left\{\zeta_{0}(r), B_{1}(r)\right\}$ also solves equation (16) by writing

$$
\begin{gathered}
B_{1}=q B_{a}+(1-q) B_{b}, B_{1}^{\prime}=q B_{a}^{\prime}+(1-q) B_{b}^{\prime}, B_{1}^{\prime \prime}=q B_{a}^{\prime \prime}+(1-q) B_{b}^{\prime \prime} \\
\text { Then } \\
B_{1}^{\prime \prime}-\frac{B_{1}^{\prime}}{r}-2 g^{2} B_{1}=q B_{a}^{\prime \prime}+(1-q) B_{b}^{\prime \prime}-\frac{q B_{a}^{\prime}}{r}-\frac{(1-q) B_{b}^{\prime}}{r} \\
\quad-2 g^{2} q B_{a}-2 g^{2}(1-q) B_{b}, \\
=q\left(B_{a}^{\prime \prime}-\frac{B_{a}^{\prime}}{r}-2 g^{2} B_{a}\right)+(1-q)\left(B_{b}^{\prime \prime}-\frac{B_{b}^{\prime}}{r}-2 g^{2} B_{b}\right)=0 .
\end{gathered}
$$

Therefore, $\left\{\zeta_{0}, q B_{a}+(1-q) B_{b}\right\}$ is a perfect fluid sphere.

\section{The New Theorem of Two Linking Theorems}

The solution generating theorem we shall present is slightly different from those developed so far. We can also simultaneously apply $T_{7}$ and $T_{8}$. So, the transformation can be represented as:

Theorem 3. Let $\zeta_{0}(r)$ and $B_{0}(r)$ be changed to $\frac{1}{\zeta_{0}(r)}$ and $B_{0}(r) Z_{0}(r)$ respectively. Define

$$
Z_{0}(r)=\left\{\sigma+\varepsilon \int \frac{r \mathrm{~d} r}{B_{0}(r)^{2}}\right\}
$$

for arbitrary $\sigma$ and $\varepsilon$. The transformation can be represented:

$$
T_{9}:\left\{\zeta_{0}(r), B_{0}(r)\right\} \text { a }\left\{\zeta_{0}^{-1}(r), B_{0}(r) Z_{0}(r)\right\},
$$

map perfect fluid spheres into perfect fluid spheres.

We can see that term ${ }^{Z_{0}}(r)$ has no change. Now, we verify

$$
T_{9} \mathrm{o} T_{9}:\left\{\zeta_{0}, B_{0}\right\} \text { a }\left\{\frac{1}{\zeta_{0}}, B_{0} Z_{0}\right\} \text { a }\left\{\zeta_{0}, B_{0} Z_{0} Z_{1}\right\}
$$

When we apply $T_{9}$ once, $\zeta(r)$ perpetually changes. Therefore, after applying $T_{9}$ n-times, the term $\zeta_{0}(r)$ has a result equivalent to $T_{7}$.

\section{THE TOV EQUATION}

Karl Schwarzschild was the physicist who found and published the first exact solution in 1916. This solution is called as the "Schwarzschild solution", which describes the gravitational field outside a static spherically symmetric object. Therefore, the Schwarzschild solution is a useful approximation for describing slowly rotating astronomical objects such as many stars and planets.

The Tolman-Oppenheimer-Volkov equation attempts to find the solution inside a static spherically symmetric object. In order for the solution to have continuity within the surface, the TOV equation explains the interior structure of static perfect fluid spheres.

\section{A. TOV Equation in Isotropic Coordinates}

The solution can be written in the form of spacetime ( $\mathrm{ds}^{2}$ ). In this current study, the solution can be expressed using the TOV equation. The TOV equation for isotropic coordinates can be represented by $[8,10]$ :

$$
\mathrm{d} s^{2}=-\zeta(r)^{2} \mathrm{~d} t^{2}+\frac{1}{\zeta(r)^{2} B(r)^{2}}\left\{\mathrm{~d} r^{2}+r^{2} \mathrm{~d} \Omega^{2}\right\}
$$

In the same way as the Schwarzschild metric [3-4]:

$$
\frac{\mathrm{d} p}{\mathrm{~d} r}=-\frac{\zeta(r)^{\prime}}{\zeta(r)}(\rho+p)
$$

$$
\text { we can set } \begin{aligned}
\zeta(r)=\exp \left(\int g(r) \mathrm{d} r\right) \text {. Hence, directly } \\
\frac{\mathrm{d} p(r)}{\mathrm{d} r}=-\frac{[\rho(r)+p(r)]\left[m(r)+4 \pi p(r) r^{3}\right]}{r^{2}[1-2 m(r) / r]} ;
\end{aligned}
$$

$$
\frac{\mathrm{d} m(r)}{\mathrm{d} r}=4 \pi \rho(r) r^{2}
$$

This equation, called the "TOV equation", describes the internal structure of general relativistic static perfect fluid spheres. The significance of the TOV equation is associated with the constraints in studying the interior structures.

We can see it gives the same result as the result attained using the Schwarzschild metric. Since the TOV equation describes the interior of stars in terms of pressure and density, they are considered as the real measures. Regardless of the changing coordinates, the form of the TOV equation is not different. 


\section{B. The Main Theorem of the TOV Equation}

Having now found the solution generating theorems in isotropic coordinates, we can then derive these theorems directly in terms of pressure and density profiles, $p(r)$ and $\rho(r)$, which are useful in generating the interior solutions for perfect fluid spheres $[8,10]$.

Theorem 4. (P1) Let $p_{0}(r)$ and $\rho_{0}(r)$ solve the TOV equation, and hold $m_{0}(r)=4 \pi \int \rho_{0}(r) r^{2} \mathrm{~d} r$ fixed. Defines an auxiliary function $g_{0}(r)$ by

$$
g_{0}(r)=\frac{m_{0}(r)+4 \pi p_{0}(r) r^{3}}{r^{2}\left[1-2 m_{0}(r) / r\right]}
$$

Then the general solution to the TOV equation is given by $p(r)=p_{0}(r)+\delta p(r)$, where

$$
\delta p(r)=\frac{\delta p_{c} \sqrt{1-2 m_{0} / r} \exp \left\{-2 \int_{0}^{r} g_{0} \mathrm{~d} r\right\}}{1+4 \pi \delta p_{c} \int_{0}^{r} \frac{1}{\sqrt{1-2 m_{0} / r}} \exp \left\{-2 \int_{0}^{r} g_{0} d r\right\} r \mathrm{~d} r},
$$

and $\delta p_{c}$ is the shift in the central pressure.

Theorem 5. (P2) Let $p_{0}(r)$ and $\rho_{0}(r)$ solve the TOV equation, and hold $g_{0}(r)$ fixed, in the sense that

$$
g_{0}(r)=\frac{m_{0}(r)+4 \pi p_{0}(r) r^{3}}{r^{2}\left[1-2 m_{0}(r) / r\right]}=\frac{m(r)+4 \pi p(r) r^{3}}{r^{2}[1-2 m(r) / r]} .
$$

Then the general solution to the TOV equation is given by $p(r)=p_{0}(r)+\delta p(r)$ and $m(r)=m_{0}(r)+\delta m(r)$, where

$$
\delta m(r)=\frac{4 \pi r^{3} \delta \rho_{c}}{3\left[1+r g_{0}\right]^{2}} \exp \left\{2 \int_{0}^{r} g_{0} \frac{1-r g_{0}}{1+r g_{0}} \mathrm{~d} r\right\}
$$

and

$$
\delta p(r)=-\frac{\delta m}{4 \pi r^{3}} \frac{1+8 \pi p_{0} r^{2}}{1-2 m_{0} / r}
$$

Here, $\delta \rho_{c}$ is the shift in the central density. Explicitly combining these formulae, we have

$$
\delta p(r)=\frac{\delta p_{c}}{\left[1+r g_{0}\right]^{2}} \frac{1+8 \pi p_{0} r^{2}}{1-2 m_{0} / r} \exp \left\{2 \int_{0}^{r} g_{0} \frac{1-r g_{0}}{1+r g_{0}} \mathrm{~d} r\right\}
$$

Next, we shall introduce a new modified theorem for the TOV equation.

\section{The modified TOV equation}

Theorem 6. Let $p_{0}(r)$ and $\rho_{0}(r)$ solve the TOV equation, and hold $g_{0}(r)$ fixed, in the sense that

$$
g_{0}(r)=\frac{m_{0}(r)+4 \pi p_{0}(r) r^{3}}{r^{2}\left[1-2 m_{0}(r) / r\right]}=\frac{m(r)+4 \pi p(r) r^{3}}{r^{2}[1-2 m(r) / r]} .
$$

Then the general solution to the TOV equation is given by $p(r)=Z_{0}(r) p_{0}(r), \rho(r)=A_{0}(r) \rho_{0}(r)$, and $m(r)=B_{0}(r) m_{0}(r)$. We define functions $Z_{0}(r)$ and $A_{0}(r)$ by

$$
Z_{0}(r)=-e^{-v(r)} \int e^{v(r)} \frac{A_{0}(r) \rho_{0}(r) g_{0}(r)}{p_{0}(r)} \mathrm{d} r
$$

where

$$
v(r)=-\int \frac{A_{0}(r) \rho_{0}(r) g_{0}(r)}{p_{0}(r)} \mathrm{d} r, \text { and }
$$

$$
A_{0}(r)=B_{0}(r)+\frac{B_{0}^{\prime}(r) m_{0}(r)}{4 \pi \rho_{0}(r) r^{2}},
$$

for all arbitrary $B_{0}(r)$.

Proof for Theorem 6. From the TOV equation

$$
\frac{\mathrm{d} p(r)}{\mathrm{d} r}=-\frac{[\rho(r)+p(r)]\left[m(r)+4 \pi p(r) r^{3}\right]}{r^{2}[1-2 m(r) / r]}
$$

Then we substitute $p(r)=Z_{0}(r) p_{0}(r), \rho(r)=A_{0}(r) \rho_{0}(r)$, and $m(r)=B_{0}(r) m_{0}(r)$ into equation (31) to give

$$
\begin{gathered}
Z_{0}(r) \frac{\mathrm{d} p_{0}(r)}{\mathrm{d} r}+\frac{\mathrm{d} Z_{0}(r)}{\mathrm{d} r} p_{0}(r)=-\frac{\left[\mathrm{A}_{0}(r) \rho_{0}(r)+Z_{0}(r) p_{0}(r)\right]\left[B_{0}(r) m_{0}(r)+4 \pi Z_{0}(r) p_{0}(r) r^{3}\right]}{r^{2}\left[1-2 B_{0}(r) m_{0}(r) / r\right]} \\
-Z_{0}(r) \frac{\left[\rho_{0}(r)+p_{0}(r)\right]\left[m_{0}(r)+4 \pi p_{0}(r) r^{3}\right]}{r^{2}\left[1-2 m_{0}(r) / r\right]}+\frac{\mathrm{d} Z_{0}(r)}{\mathrm{d} r} p_{0}(r)= \\
-\frac{\left[A_{0}(r) \rho_{0}(r)+Z_{0}(r) p_{0}(r)\right]\left[B_{0}(r) m_{0}(r)+4 \pi Z_{0}(r) p_{0}(r) r^{3}\right]}{r^{2}\left[1-2 B_{0}(r) m_{0}(r) / r\right]} \\
-Z_{0}(r)\left[\rho_{0}(r)+p_{0}(r)\right] g_{0}(r)+\frac{\mathrm{d} Z_{0}(r)}{\mathrm{d} r} p_{0}(r)=-\left[A_{0}(r) \rho_{0}(r)+Z_{0}(r) p_{0}(r)\right] g_{0}(r)
\end{gathered}
$$

So, when we get the first order linear non-homogeneous equation in terms of $Z_{0}(r)$, we can solve for $Z_{0}(r)$ by integrating factor method. Finally,

$$
Z_{0}(r)=-e^{-v(r)} \int e^{v(r)} \frac{A_{0}(r) \rho_{0}(r) g_{0}(r)}{p_{0}(r)} \mathrm{d} r,
$$




$$
\text { where } v(r)=-\int \frac{A_{0}(r) \rho_{0}(r) g_{0}(r)}{p_{0}(r)} \mathrm{d} r \text {. }
$$

Next, we consider $\frac{\mathrm{d} m(r)}{\mathrm{d} r}=4 \pi \rho(r) r^{2}$. gives;

The extension of $m(r)$ and $\rho(r)$, in a more suggestive form

$$
A_{0}(r)=B_{0}(r)+\frac{B_{0}^{\prime}(r) m_{0}(r)}{4 \pi \rho_{0}(r) r^{2}}
$$

\section{CONCLUSION}

Generally, we have been generating new several solutions using an assumption of perfect fluid spheres. In this paper, we focus on isotropic coordinates for extending class of new several solutions. We analyzed the relationship between the solution generating theorems that map perfect fluid spheres into perfect fluid spheres. These theorems lead us to a new corollary and some additional properties. Moreover, we deform these solutions into the TOV equation in terms of pressure and density and develop a new modified theorem for the TOV equation.

\section{ACKNOWLEDGMENT}

This project was funded by the Ratchadapisek Sompoch Endowment Fund, Chulalongkorn University. AK was supported by the Science Achievement Scholarship of Thailand (SAST). PB was additionally supported by a scholarship from the Royal Government of Thailand.

\section{REFERENCES}

[1] R. M. Wald, “General relativity”, University of Chicago Press, 1984.

[2] C. W. Misner, K. S. Thorne, and J. A. Wheeler, “Gravitation”, W. H. Freeman and Company, San Francisco, 1973.

[3] P. Karndumri, “General relativity”, Danex intercorporation Co., Ltd., Bangkok, 2015.

[4] S. M. Carrol, "Spacetime and geometry : an introduction to general relativity”, Addison-Wesley, San Francisco, USA, 2003.

[5] M. R. Finch and J. E. F. Skea, "A review of the relativistic static fluid sphere”, 1998.

[6] P. Boonserm and M. Visser, "Buchdahl-like transformations for perfect fluid spheres”, Int. J. Mod. Phys. D17, 2008.

[7] P. Boonserm, M. Visser, and S. Weinfurtner, "Generating perfect fluid spheres in general relativity”, Phys. Rev. D71.124037, 2005.

[8] P. Boonserm, "Some exact solutions in general relativity”, MSc. Thesis, Victoria University of Wellington, 2006.

[9] K. Thairatana, "Transformation for perfect fluid spheres in isotropic coordinates”, MSc. Thesis, Chulalongkorn, 2011.

[10] P. Boonserm, M. Visser, and S. Weinfurtner, "Solution generating theorems for the TOV equation”, Phys. Rev. D76, 2007.

[11] P. Boonserm, P. Suavansri, and K. Thairatana, "Transformation for perfect fluid spheres in isotropic coordinates”, [Annual Meeting in Mathematics (AMM2011), Khon Kaen, Thailand, pp. 261-272, 10-11 March, 2011]. 